\title{
An Ergodic-Theoretical Approach to the Chaotic Behaviour of Dynamical Systems
}

\author{
By \\ Yoichiro TAKAHASHI*
}

\section{§. Introduction}

In the present paper we shall survey a series of results concerning the chaotic behaviours of one dimensional maps from an ergodic theoretical aspect, which has a far origin in a meeting on the topological entropy held at RIMS a little more than ten years ago.

In recent years the chaotic behaviour of dynamical systems is a common concern not only among various branches of mathematics but also among various branches of sciences and engineerings. For instance, statistical physicists seem to have accepted it as one of the general behaviours in nonequilibrium statistical mechanics. Especially, Feigenbaum's and other critical phenomena in bifurcation diagrams of dynamical systems are intensively studied among them.

As to the statistical mechanics the close relationship was established in 1970's between ergodic theory and equilibrium statistical mechanics since Sinai [22]. One may say that the theory of Axiom A diffeomorphisms and expanding maps is "isomorphic" to the theory of the equilibrium statistical mechanics of one dimensional lattice systems and the introduction of the notion of Gibbs measures has brought several deep results such as [3].

The current attention to the chaotic behaviour may be said to have started with the papers of Ruelle-Takens [21], May [14], Li-Yorke [11], Feigenbaum [34] et al. The Ueda attractor called Japanese attractor by D. Ruelle for the periodically forced Duffing equation was already observed by Y. Ueda in 1960's but it did not draw any attention till recent years ([33]). The existence of the complicated behaviours of simple dynamical systems in itself was known since

Received March 9, 1983.

* Department of Mathematics, College of General Education, The University of Tokyo, Komaba, Meguro-ku Tokyo. Japan. 
Poincaré for individual differential equations in low dimension.

One of the goals of mathematical study of the chaotic behaviour is to establish the global bifurcation diagram for the family of dynamical systems. There are many works on the bifurcation of dynamical systems but the bifurcation theory is a local theory and still there are difficulties to obtain the global feature. A most hopeful aspect may be the proposal in [7] but we confine ourselves to mention a recent observation by $\mathrm{H}$. Oka et al. on the famous Lorenz equation that the differential equations with attractors of Lorenz type form a corn-like region with vertex at the origin in the versal family at $\left(\begin{array}{lll}0 & 1 & 0 \\ 0 & 0 & 0 \\ 0 & 0 & 0\end{array}\right)$. In other words, they observed the "germ" of the strange attractors at the degenerated singular point. It is also an interesting problem to study the bifurcation diagram restricted only to stable periodic orbits. The numerical results of Y. Aizawa suggests that the existence of a strange attractor might be deduced from verifying that the dynamical system lies on a path combining two dynamical systems with stable periodic orbits of different topological type ([1]).

Our main concern lies in the rough classification of chaotic behaviours of dynamical systems. We employ the classification of the topologically chaotic systems: observable chaos and latent chaos. The latent one corresponds to the phenomenon called window by May. It takes place, for example, under the coexistence of a stable periodic orbit and a repeller which is a Cantor set. The classification was first given in [18] and the definition will be found in Section 2. The importance of our classification may be explained as follows. On one hand, the window phenomenon corresponds to the situations where chaos is not observed by numerical (and, probably, real) experiments for systems which are verified to have topologically chaotic behaviour. (Precisely to say, the latent chaos may be observed as a transient phenomenon.) For instance, there is a route of the transition to chaos called phase locking. It starts with quasiperiodic motion, then a stable periodic motion is observed with a constant period (phase locking) and finally the chaotic behaviour follows suddenly. This procedure should be understood as the birth and growth of the latent chaos under the presence of stable periodic orbits. (Compare with [35] p. 250.) On the other hand, our classification is comparable with that of phases in thermodynamics (gas, solid etc.). In fact, in some special cases the correspondence is rigorously done by the "isomorphism" between the dynamical systems and the lattice systems. But we confine ourselves in the present paper to survey mathematical results 
concerning the classification and its characterization for one dimensional maps and their origins in traditional ergodic theory.

Finally we shall mention a few words on the external noise and the random family. The responce of chaos against external random noise is, of course, an important topic, and which can be treated in some aspect by generalizing the method stated in Section 3 (cf. [18]). It may be worthy to mention I. Tsuda's observation by numerical experiment on a map of an interval related to the Belousov-Zhabotinskii reaction: as the size of the external random noise increases, the value of the characteristic exponent decreases and the periodic ordered motion is verified ([13]). In the other direction it will be important to study the random family of dynamical systems ([16]). It is shown in [9] that there is a bifurcation of bands or islands (cf. $\S 2$ for the definition) which is different from that for a single dynamical system.

\section{§1. Number-Theoretical Transformations}

Number theory is one of the branches which furnish basic examples to ergodic theory. We would like to survey several results on the numbertheoretical transformations in order to clarify the origins of the results which will be stated in the proceeding sections.

Traditionally much attention has been paid to the expansion $t=\rho(x)$ of a real number $t$ in terms of an integer sequence $x=x(t)\left(x_{n}\right)$ such as the continued fractional expansion

$$
t=\rho(x) \equiv \frac{1}{x_{0}}+\frac{1}{x_{1}}+\frac{1}{x_{2}}+\cdots
$$

and the binary expansion

$$
t=\rho(x) \equiv 2^{-1} x_{0}+2^{-2} x_{1}+\cdots .
$$

Here we are concerned with real numbers in the unit interval $I$. Each expansion admits an associated map $f$ of $I$ to itself. For example,

$$
f(t)=\left\{\frac{1}{t}\right\} \text { and }\{2 t\},
$$

respectively, in cases (1.1) and (1.2), where $\{\cdot\}$ stands for the fractional part of a real number. The coefficient $x_{n}=x_{n}(t)$ of the expansion are determined by the orbit $f^{n} t, n \geqq 0$, under the map $f$ as

$$
x_{n}(t)=x_{0}\left(f^{n} t\right)
$$


and the function $x_{0}(t)$ corresponds to a numbering of the minimal partition $A=\{a\}$ of the unit interval $I$ such that $f$ is monotone on each atom $a$. It then turns out that the expansion such as (1.1) and (1.2) is equivalent to the expression

$$
t=\rho(x)=f_{x_{0}}^{-1} \circ f_{x_{1}}^{-1} \circ \cdots=\lim _{n \rightarrow \infty}\left(f_{x_{0}}^{-1} \circ \cdots \circ f_{x_{n}}^{-1}\right)\left(t^{\prime}\right), \quad x=x(t),
$$

for an arbitrary $t^{\prime}$ where $f_{a}^{-1}$ is the inverse map of $f$ on the atom $a$ (identified with the subinterval $\left.I_{a}=\left\{t ; x_{0}(t)=a\right\}\right)$.

Conversely, let us given a piecewise continuous map $f$ first. For example,

$$
f(t)=\{\beta t\} \quad t \in I=[0,1) \quad(\beta>1) .
$$

This map is called $\beta$-transformation. It is quite natural to ask whether there exists the expansion (1.4) associated with $f$. The traditional interest had lain on the expanding maps since, otherwise, (1.4) may lose its meaning (cf. § 2). When

$$
\text { ess. inf }\left|f^{\prime}\right| \equiv c>1 \text {, }
$$

the existence of the limit (1.4) follows immediately, e.g., by solving the functional equation

$$
\rho(x)=\widetilde{f_{x_{0}}^{-1}}(\rho(S x))
$$

on the product space $A^{\boldsymbol{N}}$ where $S$ is the shift, i.e.,

$$
S x=\left(x_{n+1}\right) \quad \text { if } \quad x=\left(x_{n}\right),
$$

$\widetilde{f_{a}^{-1}}$ is a suitable extension of $f_{a}^{-1}$ which has $I$ as its domain and the set $A$ is defined as before. It is also easy to obtain the following

Theorem 1.1. Assume (1.6). Then there exists a closed subset $X=X(f)$ which is invariant under $S, S X=X$, such that

(i) $\rho: X \rightarrow I$ is a continuous surjection and is injective except possibly for countably many points in $X$ where it is 2 to 1 ,

(ii) $f(\rho(x))=\rho(S x) \quad$ for $x \in X$.

The proof is omitted here since it is a special case of the general theorem stated in Section 2 but we state here only that

$$
X=\left\{x \in A^{N} ; \bigcap_{i=0}^{n} f^{-i}\left(\text { int } I_{x_{i}}\right) \neq \varnothing \quad(n \geqq 0)\right\} .
$$

The triplet $(X, S, \rho)$ is called the realization of $f$ and the analysis of the dynamical system $(I, f)$ can be reduced to that of the realization.

The structure of the set $X=X(f)$, which will be stated as Theorems 2.4-5 
in Section 2 in the general situation, was investigated in $[10,25,26]$ for $\beta$ transformation and the result can be rephrased as follows:

Theorem 1.2. Let $\beta>1$ and $f$ be defined by (1.5). Then the followings hold:

(i) $X=\left\{x \in\{0, \ldots, N-1\}^{N} ; S^{n} x \geqq x_{\beta}^{*}\right\}$ with $x_{\beta}^{*}=\lim _{t \rightarrow 1} x(t)$

where $\leqq$ denotes the lexicographical order and $N$ is the integer such that $N-1<\beta \leqq N$.

(ii) The sequence $x_{\beta}^{*}$ is monotone increasing in $\beta$ w.r.t. the lexicographical order.

(iii) Let us denote the fixed point set by Fix (.) and put

$$
D_{0}(z)=\exp \left\{-\sum_{n=1}^{\infty} \frac{z^{n}}{n} \# \operatorname{Fix}\left(I, f^{n}\right)\right\} \text {. }
$$

This formal power series in $z$ is the reciprocal of the Artin-Mazur zeta function. Then,

$$
D_{0}(z)=1-\sum_{n=0}^{\infty} a_{n} z^{n+1} \quad \text { where } \quad\left(a_{n}\right)=x_{\beta}^{*} .
$$

(iv) $\rho(x)=\sum_{n=0}^{\infty} x_{n} \beta^{-n-1} \quad(x \in X)$.

(v) The topological entropy of $(X, S)$ is $h(X, S)=\log \beta$.

From the ergodic theoretical point of view we are now to find the natural invariant measure for $f$ on $I$ or for $S$ on $X$. In our context absolutely continuous measures on $I$ have an a priori meaning and so the following operator was introduced in [20]:

$$
\mathscr{H} u(t)=\sum_{s \in f^{-1} t} u(s) .
$$

Exactly to say, the dynamics on $L^{1}(I, d t)$ induced by $f$ is governed by the operator $\mathscr{L}=\beta^{-1} \mathscr{} \mathscr{}$, which is generally defined as

$$
\mathscr{L} u(t)=\sum_{s \in f^{-1} t} u(s) /\left|f^{\prime}(s)\right|
$$

and is now called Perron-Frobenius operator.

Put

$$
D_{1}(z)=\exp \left[-\sum_{n=1}^{\infty} \frac{z^{n}}{n} Q_{n}(f)\right], \quad Q_{n}(f)=\sum_{t \in \mathrm{Fix}\left(f^{n}\right)} \frac{1}{\left|\left(f^{n}\right)^{\prime}(t)\right|} .
$$

Then the method and the results in $[10,25,26]$ can be rephrased as follows: 
Theorem 1.3. Let $f$ be the $\beta$-transformation. Then, the followings hold:

(i) $D_{1}(z)=D_{0}(z / \beta)=1-\sum_{n=0}^{\infty} a_{n} z^{n+1} / \beta^{n+1}$

(ii) $z=1$ is the unique zero with the smallest absolute value and it is an isolated simple zero.

(iii) There is a unique solution $u=u^{*}$ of the eigenvalue problem with unit $\boldsymbol{L}^{1}$-norm

$$
\mathscr{L} u=u
$$

and therefore it defines an absolutely continuous invariant measure $\mu^{*}$ of $f$.

(iv) The endomorphism $\left(I, f, \mu^{*}\right)$ is exact and satisfies the uniform mixing condition. In particular, it is weak Bernoulli.

(v) The natural extension of $\left(I, f, \mu^{*}\right)$ is an automorphism which is isomorphic to a Markov automorphism with possibly countable alphabet set.

(vi) The invariant probability measure $\mu^{*}$ is characterized by the variational principle

$$
\max \left\{h_{\mu}(I, f)-\log \beta\right\}=h_{\mu^{*}}(I, f)-\log \beta=0
$$

where the maximum is taken over all the invariant probability measures $\mu$ for $f$.

Remark. The invariant density function $u^{*}$ is given by

$$
u^{*}(t)=C^{-1} \sum_{n=0}^{\infty} \beta^{-n} u_{n}(t)
$$

where $C$ is the normalizing constant and

$$
u_{n}(t)=1\left(t<f^{n} 1\right) \quad \text { with } \quad f^{n} 1=\lim _{t \rightarrow 1} f^{n} t \quad(n \geqq 0),
$$

where $1(\cdot)$ is the indicator function. Generally, if $z \in C$ and $|z|<\beta$, then the eigenfunction of

$$
u=z \mathscr{L} u
$$

exists iff $D_{1}(z)=0$. The eigenfunction is then proportional to

$$
\sum_{n=0}^{\infty} \beta^{-n-1} z^{n+1} u_{n}(t)
$$

\section{§2. Unimodal Linear Transformations}

Let $a$ and $b$ positive real numbers such that 


$$
s(a, b) \equiv a+b \geqq 1 .
$$

The following map of the unit closed interval $I=[0,1]$ to itself is called a unimodal linear transformation:

$$
f(t)=f_{a, b}(t)= \begin{cases}(t+a+b-1) / b & \text { if } t \in I_{0}=[0,1-a] \\ (1-t) / a & \text { if } t \in I_{1}=[1-a, 1] .\end{cases}
$$

The family of maps, $U M L=\left\{f_{a, b} ; a>0, b>0, s(a, b) \geqq 1\right\}$ is studied in detail by S. Ito, H. Nakada and S. Tanaka in [8] and it is the first nontrivial example for which the global bifurcation diagram is obtained not only as topological dynamics but also from the ergodic-theoretical point of view. We would like to sketch several important results on $U M L$.

In the following we shall assume

$$
0<a<1 \text { and } a b<1
$$

since, otherwise the $f$-orbits are trivial in the sense that they converge to the fixed point $q=1 /(1+a)$ or the periodic orbit of period 2 or they have period 4 . On the other hand, if (2.3) holds there are infinitely many periodic points. In other words, by the piecewise linearity, the period doubling procedure is collapsed at period 4 in the Šarkovskii order for the coexistence of periods [23]:

$$
\begin{aligned}
& 3 \vdash 5 \vdash 7 \vdash 9 \vdash 11 \vdash \cdots \vdash 2 \cdot 3 \vdash 2 \cdot 5 \vdash 2 \cdot 7 \vdash 2 \cdot 9 \vdash \cdots \\
& \cdots \vdash 2^{m} \cdot 3 \vdash 2^{m} \cdot 5 \vdash 2^{m} \cdot 7 \vdash \cdots \\
& \cdots \vdash 2^{4} \vdash 2^{3} \vdash 2^{2} \vdash 2 \vdash 1
\end{aligned}
$$

But, in virtue of the piecewise linearity, the family $U M L$ has a nice property that the period doubling transformations, $T_{+}$and $T_{-}$below, perserve $U M L$ :

Lemma. Assume that $a(a+b) \geqq 1$.

(i ) Let $q_{-}=f_{a, b}^{2}(0)$ and $q_{+}=f_{a, b}^{2}(1)=f_{a, b}(0)$. Then, $f_{a, b}\left[0, q_{-}\right]=\left[q_{+}, 1\right]$ and $f_{a, b}\left[q_{+}, 1\right]=\left[0, q_{-}\right]$.

(ii) In particular, there are no periodic orbit of odd period greater than 1 under $f_{a, b}$.

(iii) Let us define transformations $T_{+}$and $T_{-}$on UML by

$$
\begin{aligned}
& T_{+}\left(f_{a, b}\right)(t)=f_{a, b}^{2}\left(q_{+}+t\left(1-q_{+}\right)\right) /\left(1-q_{+}\right), \\
& T_{-}\left(f_{a, b}\right)(t)=1-f_{a, b}^{2}\left((1-t) q_{-}\right) / q_{-} .
\end{aligned}
$$

Then,

$$
T_{+}\left(f_{a, b}\right)=T_{-}\left(f_{a, b}\right)=f_{a b, a^{2}} .
$$


Proof. Obvious.

On the other hand, if

$$
a(a+b)<1,
$$

there exists a snap-back repeller. In fact, $q=1 /(1+a)$ is then an unstable fixed point and a small neighbourhood $W$ of the point $\left(f_{a, b}\right)_{0}^{-1}(q)=\left(1-a^{2}-a b\right) /(1+a)$ satisfies $f_{a, b}^{2}(W) \supset f_{a, b}(W) \ni q$ and $f_{a, b}^{n}(W) \supset W$ for some $n \geqq 1$. Hence it follows from the intermediate value theorem that there exist periodic orbits with arbitrary sufficiently long periods. Moreover, $f_{a, b}$ shows chaos in the sense of Li-Yorke [11]. But we prefer the following definition of chaos on the topological level:

Definition 1. A dynamical system $(I, f)$ is said to show formal chaos if some iterate $\left(I, f^{p}\right), p \geqq 1$, contains a subsystem which is conjugate to the full 2-shift $\left(\{0,1\}^{N}, S\right)$.

Thus, the condition (2.6) implies formal chaos and formal chaos implies the positivity of the topological entropy.

Now let us pick up the emergence or disappearance curve in $U M L$ for some periods:

Theorem $2.1([8])$. (i) The map $f_{a, b}$ has no periodic orbit of period $2^{m} k$ with $k$ odd $\geqq 3$ and $m \geqq 0$ iff

$$
a^{i_{m+1}} b^{i_{m}}(a+b) \geqq 1
$$

where $i_{m}=\left[2^{m+1} / 3\right]$.

(ii) The map $f_{a, b}$ has a periodic orbit of odd period $k \geqq 3$ iff

$$
1-a \geqq b\left(a-a^{2}+a^{3}-\cdots+a^{k-2}\right) .
$$

The assertion (ii) follows from the direct computation based on the existence of the oscillating periodic orbit

$$
f^{k-1} t<\cdots<f^{2} t<t=f^{k} t<f^{3} t<\cdots<f^{k-2} t .
$$

Combining (ii) and Lemma, one obtains the condition for the existence of period $2^{m} k$ with odd $k$.

For a while let us consider the property of the map $T:(a, b) \mapsto\left(a b, a^{2}\right)$ defined on the set

$$
E_{0}=\{(a, b) ; 1 \geqq a \geqq 0, b \geqq 0, a b \leqq 1, s(T(a, b)) \geqq 1\}
$$

with image 


$$
E \equiv T\left(E_{0}\right)=\{(a, b) ; 1 \geqq a, b \geqq 0, s(a, b) \geqq 1\} .
$$

The map $T$ has the unique fixed point $(1,1)$ and the jacobian matrix $\left[\begin{array}{ll}1 & 1 \\ 2 & 0\end{array}\right]$ has eigenvalues 2 and -1 . This fixed point is not hyperbolic but the action of $T$ on $E$ can be regarded as hyperbolic since the unstable manifold of $(1,1)$ is the segment of the diagonal $a=b$ on which the jacobian matrix is $\left[\begin{array}{cc}a & a \\ 2 a & 0\end{array}\right]$. This fact gives rise to the following special case of Feigenbaum's critical phenomenon ([34], cf. [4]) (for "band splitting"):

Theorem 2.1. Let $f_{\mu}=f_{a(\mu), b(\mu)}, 0 \leqq \mu \leqq \mu_{\infty}=1$, be a smooth one-parameter subfamily of UML. Assume that $(a(\mu), b(\mu)) \in E_{0}, a(1)=b(1)=1$ and $\left(a^{\prime}(\mu)\right.$, $\left.b^{\prime}(\mu)\right) \neq 0$ at each point $\mu$. Then, for every sufficiently large $n$, there exists a unique $\mu_{n} \in[0,1]$ such that

(i ) $s\left(T^{n}\left(a\left(\mu_{n}\right), b\left(\mu_{n}\right)\right)=1\right.$; in other words, there is a partition of I with $2^{n}-1$ division points which is invariant under $f_{\mu_{n}}$ but there is no invariant partition finer than it,

(ii) $\mu_{\infty}-\mu_{n} \sim$ const. $\delta^{-n}$ as $n \rightarrow \infty$ with $\delta=2$,

(iii) $\lim _{n \rightarrow \infty} T_{+}^{n} f_{\mu_{n}}=\lim _{n \rightarrow \infty} T^{n} f_{\mu_{n}}=f_{1 / 2,1 / 2}$.

Remark. The map $f_{1 / 2,1 / 2}$ has several distinguished properties, e.g., it preserves the Lebesgue measure and the metrical entropy w.r.t. it is $\log 2$, the maximal value of the topological entropy among $U M L$.

The most impressive result in [8] is the discovery of window and island phenomena (see below for the definitions) in $U M L$, which indicates the ubiquity of these phenomena since the piecewise linearity usually simplifies the dynamical structure towards pure nondeterminism or determinism.

Definition 2. Let $M$ be a Riemannian manifold (with or without boundary and possibly branched) with Riemannian volume $m$ and $f: M \rightarrow M$ a $C^{1}$-map. An $f$-invariant probability Borel measure $\mu$ is called asymptotic measure on an open subset $W$ of $M$ if

$$
\lim _{n \rightarrow \infty} \frac{1}{n} \sum_{i=0}^{n-1} \delta_{f^{i} p}=\mu \quad m \text {-a.e. } p \in W
$$

where $\delta_{p}$ stands for the Dirac measure at $p$.

Definition 3. Let $(M, f)$ be as above and $W$ an open subset of $M$.

(a) $f$ is said to show observable chaos on $W$ if there exists the asymptotic measure on $W$ and the metrical entropy of $f$ w.r.t. it is positive. 
(b) $f$ is said to show island phenomenon if it shows observable chaos and the support of the asymptotic measure is not connected. The connected components are called islands.

(c) $f$ is said to show window phenomenon on $W$ if the asymptotic measure on $W$ exists and is supported by a finite set (which is necessarily a stable periodic orbit).

Remark. There is an important, critical case left unclassified above, namely, the case when there is the asymptotic measure on whose support $f$ is an almost periodic motion. Usually, the "phase locking" phenomenon starts from this case, passes (c) and ends in (a).

Theorem 2.3 ([8]). Let $k \geqq 3$ and put

$$
\begin{gathered}
W_{k}=\left\{(a, b) ; a>0, b>0,1>a\left(1+b+\cdots+b^{k-2}\right), a b^{k-1}>1\right\}, \\
I_{k}=\left\{(a, b) ; a>0, b>0,1>a\left(1+b+\cdots+b^{k-2}\right),\right. \\
\left.a b^{k-1}<1, a(a+b) b^{k-2} \geqq 1\right\} .
\end{gathered}
$$

(i) If (2.6) is satisfied and $(a, b)$ belongs to none of $W_{k}$ and $I_{k}, k \geqq 3$, then $f_{a, b}$ shows observable chaos (with one island).

(ii) For $(a, b) \in W_{k}, f_{a, b}$ shows window phenomenon on I and the stable orbit is of period $k$.

(iii) For $(a, b) \in I_{k}, f_{a, b}$ shows island phenomenon on $I$ and the number of islands is $k$ or $2 k$.

Let us illustrate Theorem 2.3 using an argument in terms of the $f$-expansion and the operator $\mathscr{L}$ introduced in the previous section. First of all, the form of the invariant density function $u^{*}(t)$ is, if any, known to be

$$
u^{*}(t)=\frac{1}{C} \sum_{n=0}^{\infty}\left(a \prod_{i=0}^{n-1} a_{i}\right) u_{n}(t)
$$

where $C$ is the normalizing constant. Let us explain the notations in (2.12). If $f^{n} t \neq 1-a$ for any $n$, then put

$$
a_{n}(t)=a_{0}\left(f^{n} t\right) \quad \text { with } \quad a_{0}(t)=\frac{1}{f^{\prime}(t)} .
$$

Then, $a_{0}(t)=b$ or $-a$ according as $t<1-a$ or not. For $s \in I$, put

$$
u_{n}^{s}(t)=1 \quad\left(t \geqq f^{n} s\right) .
$$

Then the desired quantities are defined as follows: 


$$
\begin{aligned}
& a_{n}=\lim _{t \rightarrow 0} a_{n}(t) \quad \text { (along } t \text { 's for which } a_{n}(t) \text { is well defined), } \\
& u_{n}(t)=\lim _{s \rightarrow 0} u_{n}^{s}(t) \\
& = \begin{cases}1\left(t \geqq f^{n} 0\right) & \text { if } a_{0} \cdots a_{n-1} \text { is negative } \\
1\left(t>f^{n} 0\right) & \text { otherwise. }\end{cases}
\end{aligned}
$$

Let $m$ be the Lebegue measure on $I$. It is easy to see

$$
\begin{array}{ll}
\mathscr{L} u_{n}^{s}(t)=a_{n}(s) u_{n+1}^{s}(t)+a & m-\text { a.e. } t \text { and } \\
\mathscr{L} u_{n}(t)=a_{n} u_{n+1}(t)+a u_{0}(t) & \text { for all } t
\end{array}
$$

in virtue of the definitions (2.14)-(2.15). As a formal series it follows from (2.12) and (2.17) that

$$
u^{*}(t)-\mathscr{L} u^{*}(t)=\left(1-\sum_{n=0}^{\infty} a \prod_{i=0}^{n-1} a_{i}\right) / C
$$

On the other hand, by integrating (2.16) w.r.t. $m$, one gets

$$
1-f^{n} s=a_{n}(s)\left(1-f^{n+1} s\right)+a .
$$

Consequently, we obtain the following:

Lemma. A real number $s \in I$ can be expanded as

$$
s=1-\sum_{n=0}^{\infty} a \prod_{i=0}^{n-1} a_{i}(s)
$$

if the Ljapunov characteristic exponent

$$
\begin{aligned}
\chi(s) & \equiv \limsup _{n \rightarrow \infty} \frac{1}{n} \sum_{i=0}^{n-1} \log \left|f^{\prime}\left(f^{i} s\right)\right| \\
& =-\liminf _{n \rightarrow \infty} \frac{1}{n} \sum_{i=0}^{n-1} \log \left|a_{i}(s)\right|
\end{aligned}
$$

is positive unless the f-orbit of $s$ hits $1-a$. If $\chi(s)$ is negative, then (2.20) diverges.

Remark. It can be shown that (2.20) also diverges if $\chi(s)=0$ except for some special cases.

Now the quantity

$$
\chi(0+) \equiv-\liminf _{n \rightarrow \infty} \frac{1}{n} \sum_{i=0}^{n-1} \log \left|a_{i}\right|
$$

may always be regarded as the value of the characteristic exponent at $s=0$. Similarly, the expression 


$$
0=1-\sum_{n=0}^{\infty} a \prod_{i=0}^{n-1} a_{i}
$$

may be regarded as the $f_{a, b}$-expansion of 0 .

Consequently we can conclude the followings:

(a) If the characteristic exponent $\chi(0+)$ is negative, then the expansion of $0,(2.22)$, diverges and the formal function $u^{*}(t)$ also diverges (and so there is no eigenfunction in $L^{1}(I, m)$ for the eigenvalue problem $\mathscr{L} u=u$ ).

(b) If the characteristic exponent $\chi(0+)$ is positive, the expansion of 0 converges, (2.22) holds and the formal eigenfunction $u^{*}(t)$ is the true eigenfunction. Furthermore, it can be shown that $u^{*}(t)=0$ outside of the orbit closure of 0 .

Let us note, here, that the statement in Lemma is a general result for piecewise $C^{1}$-maps: The expansion (1.4) converges for $t \in I$ such that the characteristic exponent is positive at $t$ (or at $t+0$ ). A generalization of this fact is as follows:

Theorem 2.4 ([28]). Let I be a bounded closed interval and $f: I \rightarrow I$ a surjective continuous map. Then there exist an at most countable compact set $A=A(f)$, a closed subset $X=X(f)$ which is invariant under the shift $S$ on $A^{\mathbf{N}}$ and a map $R$ of $X$ to the set of closed subintervals of $I$ with the following properties:

( $\mathrm{j})$ There is an order $<$ in $X$ w.r.t. which $R$ is monotone in the sense that $\max R(x) \leqq \min R\left(x^{\prime}\right)$ if $x<x^{\prime}$.

(ii) $R(S x) \supset f(R(x))$.

(iii) The union of $R(x), x \in X$, is $I$.

(iv) There is a subset $X_{0}$ of $X$ such that $S X_{0} \subset X_{0}$ and the complement $X_{1}=X \backslash X_{0}$ is at most countable.

(v) For $x \in X_{0}$, the interval $R(x)$ consists of a single point, say, $\rho(x)$. The map $\rho: X_{0} \rightarrow I$ is then continuous and satisfies $\rho(S x)=f(\rho(x))$.

Remark. (i) Let $t \in I$. Then $t$ belongs to the image $\rho\left(X_{0}\right)$ or does not according as the characteristic exponent is positive or negative at $t$, respectively.

(ii) The alphabet set $A$ is taken just as in Section 1 .

Finally we shall introduce several results for $U M L$ which are known for $\beta$-transformations. The first one is an extension of Theorem 1.2(i).

Theorem 2.5. The shift invariant set $X(f)$ in Theorem 2.4 is characterized 
as follows. For $a \in A$, let $\bar{x}_{a}$ and $\underline{x}_{a}$ be the maximal and the minimal among $x \in X$ such that $x_{0}=a$ w.r.t. the order introduced in Theorem 2.4(i). Then,

$$
X=\left\{x \in A^{N} ; \underline{x}_{x_{n}} \leqq S^{n} x \leqq \bar{x}_{x_{n}} \quad \text { for all } n\right\} .
$$

For a unimodal linear map, $A=\{0,1\}, \underline{x}_{1}=11 x^{*}, \bar{x}_{0}=01 x^{*}$ and $\bar{x}_{1}=1 x^{*}$ with $x^{*}=\underline{x}_{0}$.

Corollary. Let $f=f_{a, b}$ be a unimodal linear map with a less than 1 . Then,

(i) $x^{*}$ is the sequence whose $n$-th coordinate is 0 or 1 according as $a_{n}=b$ or $-a$, respectively.

(ii) $X=\left\{x \in\{0,1\}^{N} ; S^{n} x \geqq x^{*}\right.$ for all $\left.n\right\}$.

Theorem 1.2(iii) and Theorem 1.3(i) correspond to the following ([29]):

Theorem 2.6. Let $f=f_{a, b}$ be a unimodal linear map for which there are no open intervals consisting only of periodic points.

(i) Assume that $f$ does not show window phenomenon. Then,

$$
\begin{aligned}
& D_{0}(z)=D_{0}^{*}(z) \equiv 1-\sum_{n=0}^{\infty}(-1)^{x_{0}^{*}+\cdots+x_{n-1}^{*} z^{n+1}}, \\
& D_{1}(z)=D_{1}^{*}(z) \equiv 1-\sum_{n=0}^{\infty}\left(a \prod_{i=0}^{n-1} a_{i}\right) z^{n+1}
\end{aligned}
$$

(ii) Assume that $f$ shows window phenomenon and let $p$ be the period of the stable periodic orbit. Then,

$$
\begin{aligned}
& D_{0}(z)=\left(1-z^{2 p}\right) D_{0}^{*}(z) \quad \text { and } \\
& D_{1}(z)=\left(1-a^{2} b^{2 p-2} z^{2 p}\right) D_{1}^{*}(z) .
\end{aligned}
$$

(iii) The topological entropy of $f$ is $-\log z^{*}$, where $z^{*}$ is the minimal positive zero of $D_{0}^{*}(z)\left(=\right.$ that of $\left.D_{0}(z)\right)$.

Remark. (i) $a b^{p-1}>1$ in (2.27).

(ii) When there are open intervals consisting only of periodic points, the new factors in (2.26) and (2.27) should be squared under a modification of Fix (·) (cf. [29]).

\section{§3. Fredholm Theory for the Operator $\mathscr{L}$}

Let us begin with a consequence of Theorem 2.6. First of all, put

$$
P_{f}=\limsup _{n \rightarrow \infty} \frac{1}{n} \log Q_{n}(f), \quad Q_{n}(f)=\sum_{t \in \mathrm{Fix}\left(f^{n}\right)} 1 /\left|\left(f^{n}\right)^{\prime}(t)\right| .
$$


Then it follows from the definition (1.13) of the formal power series $D_{1}(z)$ that

$$
\begin{aligned}
\exp \left[-P_{f}\right] & =\min \left\{z ; z \in \boldsymbol{R}^{+}, D_{1}(z)=0\right\} \\
& =\min \left\{|z| ; z \in \boldsymbol{C}, \quad D_{1}(z)=0\right\} .
\end{aligned}
$$

Consequently, we obtain the following:

Theorem 3.1. (i) $P_{f}>0$ if $f$ has a strictly stable periodic orbit. (Hence, it shows window phenomenon.)

(ii) $P_{f} \leqq 0$ if $f$ shows observable chaos on $I$.

The proof will be found in [31] for (piecewise) local diffeomorphisms of a bounded closed interval (and, automatically, possibly branched manifold of one dimension).

Remark. (i) In the case (ii) for $U M L, P_{f}=0$ and the asymptotic measure is absolutely continuous w.r.t. the Lebesgue measure.

(ii) There are cases where $P_{f}$ is negative. For instance, consider the map

$$
g_{a, b}(t)= \begin{cases}t / a & \text { if } t \in I_{0} \equiv[0,1-b] \text { and } t \leqq a \\ 1 & \text { if } t \in I_{0} \text { and } t \geqq a \\ (1-t) / b & \text { if } t \in I_{1} \equiv[1-b, 1]\end{cases}
$$

where $a>0, b>0$, and $a+b<1$. Then it is easy to see that

$$
X\left(g_{a, b}\right)=\{0,1\}^{N}, \quad D_{0}(z)=1-2 z, \quad D_{1}(z)=1-(a+b) z .
$$

Consequently, $P_{g_{a, b}}=\log (a+b)<0$. In this case, the asymptotic measure is supported by an attractor which is a Cantor set. The Hausdorff dimension of the Cantor attractor is given for a wide class of maps as $\inf \left\{\beta ; D_{\beta}(1) \geqq 0\right\}$ where $D_{\beta}(z)$ is a formal power series interpolating $D_{0}(z)$ and $D_{1}(z)$.

A heuristic argument was given in [18] for Theorem 3.1. The quantity $Q_{n}(f)$ defined by (3.1) may formally be regarded as the trace of the operator $\mathscr{L}^{n}$ and so let us write

$$
Q_{n}(f)=\text { "tr" } " \mathscr{L}^{n}
$$

Then we may regard

$$
D_{1}(z)=\exp \left\{-\sum_{n=1}^{\infty} \frac{z^{n}}{n} \text { “tr" } \mathscr{L}^{n}\right\}=\text { "det" }(I d-z \mathscr{L}) .
$$

Recall the classical Fredholm theory for compact integral operators (cf. [15]). Then the relation $D_{1}(z)=0$ agrees with the existence of the eigenfunction of $u=z \mathscr{L} u$ and the multiplicity of the zero is related to the multiplicity of the 
eigenvalue $1 / z$, as is actually shown in Theorem 1.3 for $\beta$-transformations. Furthermore, the number of zeros on the unit circle is that of the islands $(=1$ if not), as is shown for $U M L$ in [29].

The formal argument above is, of course, far from the rigorousness.

Theorem 3.2 ([29]). Assume that $f$ is a surjective piecewise $C^{1}$-map of a bounded closed interval $I$ and is not injective. Take any complex number $z$ with $|z|<1$. Then it is an eigenvalue of $\mathscr{L}$ on the space $L^{1}(I, d i)$ with infinite multiplicity. In particular, $\mathscr{L}$ is always noncompact.

The proof follows from the facts that $\mathscr{L}$ admits a right inverse which is an isometry on $\boldsymbol{L}^{1}(I, d t)$ and that $\operatorname{dim} \operatorname{ker} \mathscr{L}=\infty$.

Nevertheless our heuristic argument has a meaning.

Theorem 3.3. Let $f$ be a piecewise linear map of a bounded closed interval I. Then there exist a disc $U$ in $C$ with center at 0 and a closed subspace $A$ of the space $\boldsymbol{B} V(I)$ of functions with bounded total variation such that

(a) $A$ is invariant under $\mathscr{L}$ and

(b) for $z \in U, D_{1}(z)=0$ iff $1 / z$ belongs to the point spectrum of the restriction of $\mathscr{L}$ to $A$, while $D_{1}(z) \neq 0$ iff $1 / z$ belongs to the resolvent.

(c) The spectral radius of $\mathscr{L}$ on $\boldsymbol{A}$ is $\exp P_{f}$.

The proof given in [29] for $U M L$ is applicable to get the theorem. We notice that, in the space $\boldsymbol{A}$, the role of Dirac measures is played by the functions which is equal to 1 at one point and 0 elsewhere.

Now let us pay attention to the special form of the formal power series $D_{0}(z)$ for $\beta$-transformations (see (1.10)). Namely, $1-D_{0}(z)$ is a power series with nonnegative integer coefficients. This property comes from a structure of the set $X(f)$, the decomposition of words in [10]. For $U M L$, it has not such a form but it turns out to be the product of such series after the decomposition of words. In [30] (cf. [31]) the notion of the shift with orbit basis is introduced for shifts $(X, S)$ for which the compactness of $X$ is not necessarily required. It is proved there that, for a shift with orbit basis,

$$
D_{0}(z)=1-\sum_{n=0}^{\infty} N_{n} z^{n}
$$

where $N_{n}$ is the number of words of length $n$ in the orbit basis.

Theorem 3.4 ([30]). If a shift $(X, S)$ can be expresses as (2.21) for some order, then every recurrent point of $(X, S)$ belongs to a subshift of $(X, S)$ 
which admits an orbit basis. In particular, the formal power series $D_{0}(z)$ is the product of power series of the form (3.6) for a piecewise continuous map of a bounded closed interval.

Now let us introduce a characterization of the quantity $P_{f}$.

Theorem 3.5 (cf. [31]). Let $f$ be a piecewise local diffeomorphism of a bounded closed interval 1 . Then, the following variational principle holds:

$$
P_{f}=\max \left\{h_{\mu}(I, f)-\int \log \left|f^{\prime}\right| d \mu\right\},
$$

where the maximum is taken over all the f-invariant probability Borel measures $\mu$.

Remark. The relation (3.7) gives the following in the case of observable chaos: $h_{\mu}(I, f)=\int \log \left|f^{\prime}\right| d \mu$ if $\mu$ is an absolutely continuous invariant probability measure. When $f$ shows window phenomenon, the asymptotic measure $\mu$ has metrical entropy 0 and so $P_{f}=-\int \log \left|f^{\prime}\right| d \mu \geqq 0$.

One of the basic lemmas for the proof of Theorem 3.5 is the following characterization of the metrical entropy of a shift invariant measure.

Lemma. Let $A$ be a finite set and $\left(A^{N}, S\right)$ the full shift over $A$. Define an operator $\mathscr{M}$ on $C\left(A^{N}\right)$ by

$$
\mathscr{U} u(x)=\sum_{a \in A} u(a . x), \quad u \in C(X), \quad a . x=\left(a, x_{0}, x_{1}, \ldots\right) .
$$

Then the following formula holds for each shift invariant probability Borel measure $\mu$ of $\left(A^{N}, S\right)$ :

$$
h_{\mu}\left(A^{N}, S\right)=\inf \left\{\int \log \frac{\mathscr{M} u(x)}{u(x)} \mu(d x)\right\}
$$

where the infimum is taken over all positive continuous functions $u$ on $A^{N}$.

Analogues to the Gibbs variational principles are studied in various situations (cf. [20] and the reference therein, also [25, 27]). Nevertheless, the intrinsic relation to the "transfer operator" such as $\mathscr{U}$ in (3.8) or (1.11) and $\mathscr{L}$ in (1.12) is not clarified but in the work of Donsker and Varadhan ([6]). In our case the quantity which appears in RHS of (3.7) is directly related to the operator $\mathscr{L}$ :

Theorem $3.6([31,32])$. Under the assumptions as in Theorem 3.5, 


$$
h_{\mu}(I, f)-\int \log \left|f^{\prime}\right| d \mu=\inf \left\{\int \log \frac{\mathscr{L} u}{u} d \mu\right\}
$$

where the infimum is taken over all positive continuous functions $u$ on the interval I.

Remark. The formula (3.10) is an extension of (3.9). There is another direction of the extension to general dynamical systems and their random perturbation ([32]). It is based upon the following fact: under the assumption as in the lemma above, let $m$ be the uniform Bernoulli measure, i.e., the product measure of the uniform measure on $A$. Then,

$$
h_{\mu}\left(A^{N}, S\right)-h_{m}\left(A^{N}, S\right)=\inf _{G} \lim \sup \frac{1}{n} \log m\left(V_{n, G}\right)
$$

where the infimum is taken over all open sets $G$ containing $\mu$ and

$$
V_{n, G}=\left\{x \in A^{N} ; \frac{1}{n}\left(\delta_{x_{0}}+\delta_{x_{1}}+\cdots+\delta_{x_{n-1}}\right) \in G\right\} .
$$

\section{References}

[1] Aizawa, Y., Statistical properties of the forced Lorenz chaos, in Random Systems and Dynamical Systems, RIMS Kokyuroku. 439 (1981), 63-88.

[2] Bowen, R., Equilibrium States and the Ergodic Theory of Anosor Diffeomorphisms, Lecture Notes in Math., 470, Springer, 1975.

[ 3 ] — Hausdorff dimension of quasi-circles, Publ. Math. IHES, 50 (1979), 11-26.

[4] Collet, P. and Eckmann, J.-P., Iterated Maps of the Interval as Dynamical Systems, Birkhauser, 1980.

[ 5 ] Cornfeld, I. P., Sinai, Ya. G. and Fomin, S. V., Ergodic Theory, Nauka, 1980 (English translation, Springer, 1981).

[6] Donsker, M. D. and Varadhan, S. R. S., Asymptotic evaluation of certain Markov process expectations for large time, I, III, Comm. Pure Appl. Math., 28 (1975), 1-45, 29 (1976), 389-461.

[7] Fujii, H., Mimura, M. and Nishiura, Y., A picture of the global bifurcation diagram in ecological and diffusion systems, Physica D, 5D (1982), 1-42.

[8] Ito, S., Nakada, H. and Tanaka, S., Unimodal linear transformations and chaos I, II, Tokyo J. Math., 2 (1979), 221-259.

[9] Ito, S. and Tanaka, S., Random iteration of unimodal linear transformations, Tokyo J. Math., 5 (1982), 463-478.

[10] Ito, Sh. and Takahashi, Y., Markov subshifts and realization of $\beta$-transformations, J. Math. Soc. Japan, 26 (1974), 33-55.

[11] Li, T. and York, J., Period three implies chaos, Amer. Math. Monthly, 82 (1975), 985-992.

[12] Lorenz, E. N., Deterministic nonperiodic flow, J. Atomos. Sci., 20 (1963), 130-141.

[13] Matsumoto, K. and Tsuda, I., Noise-induced order, J. Stat. Phys., to appear. 
[14] May, R., Biological populations obeying difference equations, stable cycles and chaos, J. Theor. Biol., 51 (1975), 511-524.

[15] Mizohata, S., Introduction to the Theory of Integral Equations, Asakura Shoten, 1968 (in Japanese).

[16] Oono, T., Ergodic properties of smooth dynamical systems with random parameters, Publ. RIMS Kyoto Univ., to appear.

[17] Oono, Y. and Osikawa, M., Chaos in $C^{0}$-endomorphisms of intervals, Publ. RIMS Kyoto Univ., 17 (1981), 165-177.

[18] Oono, Y. and Takahashi, Y., Chaos, external noise and Fredholm theory, Prog. Theor. Physics, 63 (1980), 1804-1807.

[19] Osel'edec, V. I., Multiplicative ergodic theorem. Liapunov number for dynamical systems, Trudy Moskov. Obšc., 19 (1968), 179-210.

[20] Ruelle, D., Thermodynamical Formalism, Encyclopedia of Math. 5, Addison-Wesley, 1978.

[21] Ruelle, D. and Takens, F., On the nature of turbulence, Comm. Math. Phys., 20 (1971), 167-192, 23 (1971), 343-344.

[22] Sinai, Ya. G., Gibbs measures in ergodic theory, Uspehi Mat. Nauk., 27 (1972), 21-64.

[23] Šarkovskii, A. N., Coexistence of cycles of a continuous map of a real line to itself, Ukrain. Mat. Ž., 16 (1964), 61-71 (in Russian).

[24] Štefan, P., A theorem of Šarkovskii on the existence of periodic orbits of continuous endomorphisms of the real line, Comm. Math. Phys., 54 (1977), 237-248.

[25] Takahashi, Y., $\beta$-transformations and symbolic dynamics, Proc. 2nd Japan-USSR Symp. Prob. Theory, Lecture Notes in Math., 330 (1973), 455-464.

[26] - Isomorphisms of $\beta$-automorphisms to Markov automorphisms, Osaka $J$. Math., 10 (1973), 175-184.

[27] — Application of topological entropy, RIMS Kokyuroku, 216 (1974), 82-98 (in Japanese).

[28] - A formula for topological entropy of one dimensional maps, Sci. Papers Coll. Gen. Educ. Univ. Tokyo, 30 (1980), 11-22.

[29] - Fredholm determinant of unimodal linear maps, Sci. Papers Coll. Gen. Educ. Univ. Tokyo, 31 (1981), 61-87.

[30] - - Shift with orbit basis and realization of one dimensional maps, Osaka J. Math., 20-4 (1984), to appear.

[31] — - Observable chaos and variational principle formalism for one dimensional maps, Proc. 4th Japan-USSR Symp. Prob. Theory, to appear.

[32] — Entropy functional (free energy) for dynamical systems and their random perturbations, Proc. Taniguchi Symp. on Stoch. Anal. at Katata and Kyoto, 1982, to appear.

[33] Totoki, H.. Pseudo-Markov transformation, Ann. Polonici Math., 41 (1982), to appear.

[34] Ueda, Y., Steady motions by Duffing's equation: a picture book of regular and chaotic motions, in New Approach to Nonlinear Problems in Dynamics ed. by P. J. Holmes, SIAM, (1980), 311-322.

[35] Zeeman, E. C., Bifurcation and catastrophy, Contemporary Math., 9 (1982), 207-272.

[36] Feigenbaum, M., Quantative universality for a class of nonlinear transformations, J. Stat. Phys., 19 (1978), 25-52; The universal metric properties of nonlinear transformations, J. Stat. Phys., 21 (1979), 669-706. 